\title{
Emmanuelle Zolesio
}

\section{MARIE LABORIE, UN CAS DE SOCIALISATION CHIRURGICALE RATÉE}

Résumé : Cet article décrit la trajectoire de Marie Laborie, jeune femme ayant abandonné la chirurgie pour se reconvertir en médecine générale. Le portrait ainsi reconstruit s'appuie sur des entretiens avec l'enquêtée, avec des chirurgiens l'ayant formée ainsi que sur son dossier scolaire. Il s'agit de comprendre les tensions qui ont existé entre certaines dimensions de sa socialisation primaire (familiale) et de sa socialisation secondaire (professionnelle). En pointant les "ratés " de la socialisation chirurgicale de Marie (notamment en comparant sa trajectoire aux autres candidate-s à la chirurgie ayant poursuivi leur cursus) on comprend mieux, en creux, les dispositions requises par le milieu professionnel. Marie avait certes intériorisé certaines valeurs chirurgicales (endurance physique, franc-parler) et acquis des compétences (notamment techniques) mais il lui manquait de toute évidence des dispositions socialement construites comme masculines et essentielles pour réussir dans le métier: assurance, endurance morale, répartie. Le milieu professionnel, soucieux de sa reproduction et percevant ses lacunes dispositionnelles, participe finalement de son éviction.

a « socialisation ratée », définie comme une "asymétrie complète entre réalité objective et subjective » (p. 271) est déclinée en trois cas par Peter L. Berger et Thomas Luckmann dans La construction sociale de la réalité (1966). Classiquement, elle peut survenir suite à un accident biographique (qu'il soit biologique ou social). Autre cas de figure: lorsque l'enfant rencontre plusieurs mondes hautement contradictoires pendant sa socialisation primaire, celle-ci a de fortes chances d'être « ratée » dans certains domaines au moins. C'est typiquement le cas d'un enfant des classes supérieures éduqué par une nounou des classes populaires. Ces injonctions contradictoires dans la socialisation primaire ont souvent été étudiées. Enfin, elle peut résulter d'une trop grande différence entre socialisations primaire et secondaire. Cette dernière configuration nous paraît avoir été peu étudiée, au moins à l'âge adulte. Les auteurs rappellent, de plus, que si la socialisation n'est jamais totalement réussie, les cas de ratage complet sont extrêmement rares.

Nous voudrions, au travers de l'exemple d'une trajectoire singulière, éclairer le troisième cas de socialisation ratée. En effet, étudier les « ratés» d'une socialisation secondaire, c'est aussi dégager en creux ce qu'elle aurait dû/pu être. En pointant les dispositions ayant manqué au candidat, on dit en fait celles que l'institution attend, requiert et contribue habituellement à façonner. Autrement dit, le

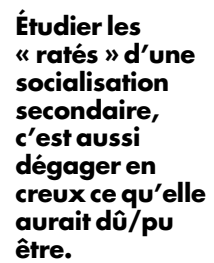


portait qui va être étudié apporte davantage que la seule compréhension d'une trajectoire singulière : il nous en apprend tout autant sur l'instance de socialisation qui a échoué dans son travail de « conversion». "Car il n'y a pas plus social, pas plus communément partagé, que les "problèmes " dits "personnels " (mauvaise conscience, incompréhension, frustration, malaise, état dépressif...) : produits des multiples décalages entre ce que nous sommes (ce que le monde social a déposé en nous) et ce que les situations exigent de nous (et que nous ne sommes pas toujours disposés à "donner »); produits des divers affrontements, confrontations ou accommodations (plus ou moins forcées) avec des personnes aux dispositions contradictoires; produits de l'inassouvissement d'inclinations constituées par le passé et qui ne trouvent pas toujours les conditions de leur expression ; produits, enfin, de l'incompatibilité des investissements dans des sphères d'activités différentes (familiales, professionnelles, amicales, culturelles...» (Lahire, 2002, p. 5).

C'est le cas de Marie Laborie ${ }^{1}$, dont la socialisation chirurgicale (la socialisation secondaire, donc) a échoué en grande partie, qu'on se propose d'étudier. Après avoir presque achevé son internat de chirurgie (elle est en cours de quatrième année ${ }^{2}$ ), elle abandonne le cursus et se réoriente vers la médecine générale. Nous l'avons rencontrée à ce moment-là, ainsi que d'autres chirurgiens ayant été témoins de son parcours. Nous avons également eu accès à son dossier scolaire. Nous nous efforcerons autant que possible de comparer la trajectoire, les réactions de Marie avec celles d'autres candidates à la chirurgie pour comprendre ce qui, dans son cas, peut permettre de parler de «socialisation ratée».

Il s'agit de se poser les questions suivantes, soulevées par les énoncés théoriques de Peter L. Berger et Thomas Luckmann : quelles peuvent être les tensions entre socialisation primaire et secondaire? En quoi la socialisation secondaire est-elle ratée? En quoi a-t-elle réussi ? Pourquoi la réalisation de l'identité subjective se heurte-t-elle aux résistances de la structure sociale?

\section{SITUER LE CAS ATYPIQUE DE MARIE LABORIE}

Avant d'aborder la trajectoire de Marie Laborie, il apparaît utile de contextualiser son cas (Glaser et Strauss, 1967) en mobilisant des données sur la place des femmes dans la carrière chirurgicale et en

1/ Il va de soi que le nom de l'enquêtée a été anonymisé, comme tous ceux des autres enquêtés cités.

2/ L’internat de chirurgie (troisième cycle des études médicales pour les spécialistes) compte cinq années au total. 
la situant par rapport aux autres trajectoires féminines étudiées dans l'enquête qualitative.

Tout d'abord, mentionnons qu'il n'y a en chirurgie digestive, spécialité finalement investie par Marie Laborie, que $10 \%$ de femmes chirurgiens $^{3}$ (contre $38 \%$ en gynécologie-obstétrique, première spécialité envisagée par l'enquêtée). Aucune statistique ne permet d'évaluer le nombre d'abandons en cours d'internat selon le sexe. Les données concernant les droits aux remords ${ }^{4}$ ne sont pas non plus sexuées et ne permettent pas d'apprécier le poids des réorientations féminines. Toutefois, ces cas de réorientations apparaissent tout à fait marginaux. Le Pr Soquet m'indique que, dans toute sa carrière sur Villedefrance, il n'a connu que deux étudiants ayant finalement abandonné la chirurgie (un homme, choisissant finalement la psychiatrie, et Marie Laborie). Par ailleurs, l'autre spécificité du cas de Marie Laborie réside dans le caractère tardif de son abandon (en quatrième année seulement, c'est-à-dire au-delà de la période tolérée pour un droit au remords, ce qui l'oblige à réviser à la baisse ses aspirations en se tournant vers le résidanat de médecine générale). Habituellement, si les femmes s'auto-excluent de la voie chirurgicale, elles le font avant l'étape décisive de l'internat.

D'autre part, toutes les autres enquêtées ayant choisi la voie de la chirurgie semblent d'une façon ou d'une autre entretenir un rapport vocationnel à la profession. Aucune n'a envisagé, même provisoirement, d'abandonner la profession ou de prendre un mi-temps professionnel pour élever ses enfants, par exemple. Seule, une autre enquêtée, qui aurait souhaité continuer dans la voie chirurgicale, se voit contrainte d'arrêter faute de poste. Profession voulue, vécue sur le mode de la vocation, elle est rarement abandonnée par les femmes qui en ont fait le choix. Une fois encore, Marie Laborie fait donc figure d'exception.

\section{UNE PETITE FILLE DES CLASSES SUPÉRIEURES}

Marie a grandi en région parisienne dans un milieu favorisé, peu enclin à remettre en cause la division traditionnelle entre les sexes. Sa socialisation primaire semble assez cohérente avec le modèle familial et valorise les dispositions socialement construites comme féminines.

\section{Sa socialisation primaire valorise les dispositions socialement construites comme féminines.}


Le père de Marie, Centralien de formation, était ingénieur dans l'aérospatial, plutôt investi et heureux dans sa profession ${ }^{5}$. Marie ne cesse de se décrire comme en admiration devant son itinéraire, quand elle était enfant. Elle attendait de sa part des encouragements qui ne sont jamais venus. Sa mère a travaillé un an ou deux comme secrétaire, mais elle s'est "empressée » (selon les mots de l'enquêtée) d'abandonner son emploi quand elle s'est mariée, pour se consacrer à l'éducation de ses enfants. Des deux côtés, les grands-mères étaient inactives alors que les grands-pères exerçaient des professions supérieures, médecin généraliste du côté paternel, commercial (avec une formation HEC) du côté maternel.

Marie est la deuxième de quatre enfants, quatre filles. Son père lui a transmis l'idée qu'il est important de faire un métier qui plaise, de préférence où l'on gagne plutôt bien sa vie mais que finalement ce second critère est moins essentiel pour une fille, son destin probable (et souhaitable) étant de se marier et le rôle du conjoint étant de subvenir aux besoins du foyer. C'est donc un schéma conjugal traditionnel qui a été inculqué. Elle se décrit d'ailleurs comme "soumise » et totalement effacée par rapport à la figure paternelle, décidant de tous les choix familiaux. Les trois sœurs de Marie exercent dans des secteurs plutôt féminins puisque deux sont infirmières et la dernière, "artiste » (elle donne quelques cours de dessin et de photo). Le parcours de Marie ne semble pas explicable par la théorie $\mathrm{du}$ « garçon manquant ${ }^{6}$, les attentes paternelles ne s'étant pas particulièrement focalisées sur elle.

Pendant toute la période du collège, Marie n'a que des amies filles. Ce n'est qu'au lycée que son entourage amical devient plus mixte. Elle n'est absolument pas « garçon manqué » mais bel et bien une «petite fille», pratiquant de la musique (flûte traversière) et faisant un peu de scoutisme, mais rejetant l'activité sportive en général. En cela, elle s'écarte des autres candidates à la chirurgie, une bonne part étant plutôt catégorisées comme "garçons manqués» du fait de leurs choix amicaux, de leur tenue vestimentaire et/ou de leur engagement sportif.

5/ Marie appartient ainsi aux $46 \%$ de médecins femmes ayant un père cadre supérieur (Breuil-Genier, Sicart, 2006)

6/ La thèse du garçon manquant soutient que les jeunes filles qui s'engagent dans des études très connotées au masculin sont porteuses d'un enjeu parental (surtout paternel) en lieu et place d'un frère manquant (dans les cas de fratries uniques ou exclusivement féminines), jouant ainsi le rôle de « fils de substitution » pour leur père. Cette hypothèse permet d'expliquer nombre de cas de femmes scientifiques québecoises (Lasvergnas, 1986) ou de trajectoires de femmes ingénieurs (Marry, 2004) ainsi que de femmes commissaires-priseurs (Quemin, 1998). Elle peut encore être mobilisée pour certains cas de femmes chirurgiens rencontrées dans le cadre de notre enquête, mais elle ne peut rendre compte de la trajectoire de Marie. 


\section{UNE TRAJECTOIRE SCOLAIRE MOYENNE COURONNÉE PAR UNE RÉUSSITE AU CONCOURS DE L'INTERNAT}

Marie fait une scolarité secondaire sans éclat, comme beaucoup d'enquêtés en chirurgie ${ }^{7}$. Elle justifie cela par un manque de travail : "J'ai toujours fait le strict minimum », "J'ai jamais été une grande bosseuse, gamine. Donc j'arrivais à passer la classe au-dessus en faisant ce qu'il fallait mais jamais en étant en tête de classe ${ }^{8}$. Elle dit avoir pensé à médecine dès son enfance, mais jugeant cette filière inaccessible vu son niveau scolaire, elle envisage plutôt d'être prof de maths. Elle obtient un bac $\mathrm{C}$, sans mention (mais sans rattrapage). Elle raconte que c'est parce qu'elle a été encouragée par l'exemple d'un copain moins bon qu'elle scolairement et qui souhaitait s'orienter vers la médecine, qu'elle a choisi également cette filière, espérant faire ses études avec lui. Il faut noter également qu'elle a plusieurs cousins et cousines plus âgés qu'elle qui ont déjà choisi avant elle la filière médicale (un cousin biologiste, une cousine généraliste, une cousine phlébologue et une cousine qui va passer l'internat en même temps qu'elle pour devenir anesthésiste), ce choix fait donc partie de l'univers des possibles.

Elle intègre une faculté de médecine parisienne, redouble sa P1 (première année de médecine, sanctionnée par un concours) comme beaucoup, et passe de justesse en troisième année. Pendant ses études de médecine, elle se met progressivement au travail et intensifie le rythme quand elle décide de préparer le concours de l'internat. Alors qu'au début de ses études de médecine, elle envisageait d'être pédiatre, au moment de la DCEM4 (sixième année de médecine, soit la dernière avant le concours de l'internat), elle ne sait plus quelle spécialité choisir. Elle s'entraîne au concours avec une amie voulant devenir gynécologue et un autre condisciple s'orientant, lui, vers la chirurgie. Par peur de se comparer aux autres, elle ne participe pas aux concours blancs, mais travaille de plus en plus intensément, "tirée " par ses camarades. En 1996, elle réussit le concours de l'internat zone Sud, classée $170^{e}$, alors qu'elle échoue au Nord à cause d'un zéro éliminatoire à une épreuve. Elle attribue essentiellement sa réussite, qu'elle n'attendait pas vraiment, à son groupe de travail : "C'est grâce à eux». Alors qu'elle décrit ses amis comme

7/ Au cours de nos travaux sur la chirurgie (dans le cadre de notre maîtrise, de notre DEA puis de notre thèse, en cours) nous avons réalisé 54 entretiens auprès de chirurgiens déjà en poste ou encore en formation (17 hommes et 37 femmes), essentiellement en chirurgie digestive.

8/ Le titre du chapitre 1 de la biographie du Pr Francine Leca, chirurgien du coeur, est assez emblématique de la trajectoire scolaire secondaire de nombre de nos enquêté-e-s: "Élève moyenne, peut mieux faire ".

\section{Contrairement aux autres enquêtées rencontrées, Marie n'a pas manifesté de véritable " vocation " pour la chirurgie, pas plus qu'elle ne s'est découverte une " passion " au cours d'un stage d'externat.}


"brillants", elle se voit d'abord comme laborieuse et ne cesse de répéter que "pour [elle] c'était inaccessible, [elle n'était] pas capable de faire ça ». Contrairement aux autres enquêtées rencontrées, Marie n'a pas manifesté de véritable « vocation » pour la chirurgie, pas plus qu'elle ne s'est découverte une «passion » pour cette spécialité au cours d'un stage d'externat et qui l'aurait portée à viser coûte que coûte un classement permettant d'envisager cette filière. Simplement, au moment des résultats, son bon classement lui permet de prétendre faire de la chirurgie à Villedefrance et c'est ce choix prestigieux qu'elle fait. Comme le montrent les travaux d'Anne-Chantal Hardy-Dubernet $(2000,2003)$, les étudiants de médecine ont en définitive aussi peu de choix quand ils sont bien classés que quand ils sont mal classés, le rang de classement agissant comme une obligation pour tous : "Les étudiants sont "affectés » d’un rang, et celui-ci témoigne d'une position hiérarchisée au sein des pairs. Le rang, c'est presque une sommation: on ne trouve pas d'étudiants «bien classés » au concours de l'internat qui choisissent l'exercice de la médecine générale " (2000, p. 93). On peut ainsi penser que l'orientation tardive de Marie va participer de l'échec ultérieur de sa socialisation secondaire.

\section{LES DÉBUTS PROMETTEURS DE LA CARRIÈRE CHIRURGICALE}

Marie choisit la chirurgie en espérant se spécialiser ensuite en gynécologie-obstétrique. Comme elle vient de se marier juste avant le début de l'internat ${ }^{9}$, et qu'elle souhaite suivre son mari qui fait son service militaire ${ }^{10}$, elle décide cependant de commencer par une année de chirurgie générale car il n'y a pas de stage de gynécologieobstétrique à proximité. Elle débute donc son internat par deux stages successifs dans le même service, d'un petit hôpital périphérique $\left(\mathrm{CHR}^{11}\right)$. Seule interne du service, elle est très proche du chef de service et opère beaucoup avec lui. "Il était content d'avoir son interne de spécialité. Lui que j'sois une fille, ça le gênait pas. " Alors qu'elle est enceinte au cours de ce stage, elle opère avec une selle ${ }^{12}$ au bloc opératoire, comme son patron d'ailleurs qui, lui, souffre d'une sciatique. Si elle acquiert une bonne technique chirurgicale de base, notamment en cœlioscopie, elle reconnaît toutefois avoir des

9/ Elle a rencontré son mari assez jeune, alors qu'elle est en deuxième année de médecine (ils habitent la même ville et faisaient du scoutisme ensemble, il a 2 ans de moins qu'elle).

10/ Lui-même a renoncé à faire son service civil pour qu'ils ne soient pas séparés.

11/ Centre Hospitalier Régional.

12/ Siège surélevé permettant d'opérer dans une position debout-assis. 
lacunes sur le plan théorique. Mais tout se passe bien et elle décrit ces semestres comme des stages "de rêve"

Après un semestre de disponibilité suite à sa grossesse, elle choisit un semestre de gynécologie-obstétrique en $\mathrm{CHU}^{13}$, cette fois. Mais Marie a eu le temps, dans ses premiers stages, d'intérioriser les jugements des chirurgiens digestifs qui lui font dire «qu’en fait les gynéco, ils savent pas très bien opérer ». Elle se juge plus habile en cœlioscopie et en vient à remettre ses premiers choix en cause: "J'(avais) pas envie d'être gynéco pour faire l'obstétrique qui me stresse et pour faire gynéco en étant un mauvais chirurgien. " Elle opte donc résolument pour la chirurgie digestive, reconnaissant ultérieurement que «c'est peut-être l'erreur (qu'elle a) faite ». À cette étape de son parcours, elle se rend bien compte qu'elle sera plus isolée en tant que membre féminin, mais n'ayant eu que des expériences positives auparavant, cela ne l'inquiète pas vraiment.

Elle poursuit avec bonheur son internat en CHU, enchaînant encore sur un stage de chirurgie digestive et sur un stage de chirurgie pédiatrique. Pour chacun de ces stages, elle obtient des appréciations positives dans ses rapports semestriels ${ }^{14}$. Elle y est décrite comme une interne consciencieuse, douée manuellement et appréciée des équipes, des patients et de leurs familles. En chirurgie pédiatrique, on lui propose même un poste d'assistant chef de clinique pour la suite, mais elle le refuse, jugeant comme une « imposture » de prendre un poste dans une spécialité qui n'est pas la sienne.

En sixième semestre, Marie choisit de faire un stage de chirurgie générale dans le service du Pr Soquet, service considéré comme «le sanctuaire de la chirurgie digestive " de Villedefrance. Ce stage sera «le stage de trop », celui qui va lui faire remettre en cause toute son orientation chirurgicale.

\section{UN STAGE DE TROP : LE CHOIX D'ARRÊTER LA CHIRURGIE}

Le service du Pr Soquet est réputé pour être un des services les plus formateurs mais aussi les plus exigeants de Villedefrance. Véritable "école de la rigueur ", il est considéré comme un passage obligé pour tous les internes de chirurgie villedefrançais. Un interne

13/ Centre Hospitalier Universitaire.

14/ Nous avons pu consulter le dossier scolaire de Marie et avons eu accès à ces comptes rendus de stages effectués par les chefs de service. 
m'explique que "c'est pas des marrants ", Marie déclare qu'ils "ont une mentalité de fous ». Si l'équipe chirurgicale compte 5 chirurgiens seniors et 4 internes, la charge de travail est telle que ces derniers sont soumis à un rythme intense. Tous rendent compte de l'énorme rythme de travail imposé (les journées commencent à $7 \mathrm{~h}$ du matin et peuvent finir à $21 \mathrm{~h}$, aux opérations s'ajoutant les gardes et les astreintes de prélèvement hépatique).

Marie choisit ce stage en sixième semestre, c'est-à-dire à un stade avancé de sa formation chirurgicale. Elle est alors enceinte de son deuxième enfant et l'annonce lorsqu'elle se présente au chef de service. Celui-ci lui dit qu'il pense que ça n'est pas une très bonne idée de venir chez lui dans ces conditions. Elle le rassure en lui disant qu'elle a mené sa première grossesse à terme et qu'elle ne compte pas prendre de congés pathologiques (elle n'en avait pas pris pour sa première). Elle m'explique en plus qu'elle pouvait être considérée comme « en surnombre » puisque le service accueillait 5 internes ce semestre-ci (avec la présence d'un interne congolais FFI ${ }^{15}$ ). A posteriori, elle justifie son choix et commente la réaction des chirurgiens du service :

« En tant qu'interne de chir, être une femme c'est déjà, c'est déjà... moins bien. On n'est déjà qu'une femme. Et c'est très macho quand même, hein. Même s'ils le disent pas c'est quand même assez macho. Et euh... Et en plus enceinte..., là ils comprenaient pas du tout que j'ai choisi ce stage-là. Pour eux c'était presque une insulte d'avoir choisi ce stage-là. Parce que c'est un des stages où on bosse le plus. Où on bosse le plus et l'avoir choisi en étant enceinte, ils ont pas compris. Alors que moi je l'avais choisi parce que justement je voulais pas y passer une fois que j'aurais mes deux enfants, je voulais y passer avant. Parce que justement, je me disais qu'après, une fois que j'aurais deux enfants, ça serait trop lourd d'avoir les gardes parce qu'ils ont des astreintes de greffe de foie là-bas ».

Marie passe un semestre très éprouvant. Physiquement, elle se sent épuisée mais elle relève le défi et fait les gardes comme ses co-internes, ne se faisant remplacer que pour les greffes (qui suivent les nuits de gardes). Elle est fréquemment humiliée en réunions de service par le Pr Soquet qui met le doigt sur ses lacunes théoriques mais elle l'accepte, reconnaissant le bien-fondé de ses critiques ("C'était mérité quelque part, hein (...) C'est dur quand même, mais bon... »).

En revanche, elle supporte très mal l'attitude du Dr Luthereau ( $\mathrm{PH}^{16}$ du service du Pr Soquet) car elle considère ses attaques comme

15/ Faisant Fonction d'Interne (grade hospitalier des étudiants étrangers de troisième cycle).

16/ Praticien hospitalier. 
des attaques personnelles, dirigées à son attention et notamment parce qu'elles sont systématiquement liées à sa grossesse: "Il me cassait mais continuellement, quoi. Il faisait tout pour me rabaisser, pour me casser ». Elle le décrit comme misogyne et raciste: "Bon, il faisait chier les Noirs et les filles, quoi, en gros (...) Puis bon, Jean-Robert (l'interne congolais) n'avait pas le même niveau non plus, c'est clair, mais je pense quand même qu'il y avait un peu de racisme là-dedans". En comparaison de son co-interne congolais, elle subit peu de remontrances de la part du Dr Luthereau pendant les opérations elles-mêmes: "Je pense que j'étais pas trop mal au bloc, je me débrouillais bien donc il avait pas trop d'occasions de me faire de réflexions". Mais celui-ci ne l'épargne pas lorsqu'elle est dans le service et elle supporte particulièrement mal ses attaques répétées, qu'elle juge injustifiées. Ses co-internes essaient de l'inciter à la rébellion :

«Eux me disaient "Mais rue dans les brancards, quoi. Rentre-lui dedans !" Et... en fait j'ai jamais voulu le faire parce que j'avais trop peur de craquer devant lui. C'est con mais... comme je prenais beaucoup sur moi et euh... que je craquais pas, que je gardais la tête froide, je me disais "Si par hasard je me mets à l'engueuler, je risque de craquer et je ne lui ferai pas ce plaisir-là !" Et donc en fait, je prenais sur moi».

En milieu de stage, un incident survient au bloc opératoire, l'antagonisme entre Marie et le Dr Luthereau atteint alors un point de non-retour :

\footnotetext{
« Le coup de crasse c'est en mi-janvier - c'est à partir de ce moment-là que je lui ai plus adressé la parole - parce que bon, cette nuit-là où je me disais "pourvu que je sois pas appelée", donc le dimanche soir où j’ai finalement passé la nuit dehors, je débute la greffe. À 8 h il y a un interne qui m'a remplacée. Les autres internes se sont dispatchés sur les autres blocs - une greffe de foie ça prend deux internes. Ça prend un chirurgien mais deux internes. Donc les autres se sont dispatchés. Et il restait Luthereau qui avait pas d'interne. Et je lui ai dit: "Ben écoute, moi je veux bien t'aider. Mais je te dis, là je suis crevée, je viens de faire la greffe [elle parle du prélèvement de foie pendant la nuit] donc il faut que tu acceptes que je prenne une selle - les selles c'est des espèces de... je sais pas si tu as vu... des espèces de sièges très hauts où on s'assoit. Avec une selle je pense que je pourrai tenir l'intervention, y a pas de souci". Et il m'a envoyée péter, en me disant: "Ouais!! Moi j'ai pas besoin d'internes qui soient capables de tenir le coup, j'ai pas besoin de... de femmes enceintes qui sont pas capables de tenir le coup, je veux une vraie interne. Je préfère encore prendre des externes", je lui dis (ton calme et posé) : "Ben vas prendre une externe". Je me suis cassée. Je suis allée dans le bureau des internes dormir un coup. Et puis voilà, quoi. Et puis bon, à partir de ce moment-là j'ai dit : "Je fais plus d'effort, c'est pas la peine, quoi". Et donc à partir de ce moment-là je lui ai plus adressé la parole. Ça allait beaucoup mieux d'ailleurs après. Parce que après il savait plus, il avait plus, il pouvait plus m'emmerder : je lui adressais plus la parole, donc il avait plus de répondant. Et donc je m'occupais plus de lui et donc ça a été mieux après du coup ».
} 
En fin de stage, elle se dit toutefois épuisée moralement par cette situation et prend quatre jours de congé pathologique :

\begin{abstract}
«J'avais pas pris du tout de vacances pour arrêter mes... pour pas prendre de congés patho et prendre mes congés annuels. Et en fait j’ai quand même pris 4 jours de congés patho parce que j'en pouvais plus, qu'ils me faisaient trop chier. Alors que physiquement je me sentais encore capable de faire 4 jours, moralement j'en avais marre et du coup j'ai arrêtée 4 jours plus tôt. C'est pas grand chose. J'ai fait mes 15 jours de vacances et j'ai enchaîné sur mes congés mater. Donc j’ai fait 5 mois au lieu de 6 . Mais j'ai été validée, hein : c'est bon, il faut 4 mois minimum pour être validé. Soquet m'a quand même dit à la fin que j'avais bien tenu le coup et qu'il en revenait pas (léger rire). Il m'a quand même fait... ma dernière semaine, donc la dernière semaine de mars, je l'ai aidé sur un pancréas. Une intervention qui a duré $7 \mathrm{~h}$. On a fait 5 h d'affilé. On s'est arrêté une demi-heure pour manger - on met un champ stérile, on s'arrête une demi-heure pour grignoter un truc, pisser. Pour pisser un coup ! Voilà, et puis on repart et on refait $2 \mathrm{~h}$. Donc on s'est arrêté, en fait on demande aux infirmières d'aller chercher à manger au self et on nous prépare des plateaux. On mange un petit truc vite fait, on boit un coup, on fait pipi et on repart. Ce qui évite de faire $7 \mathrm{~h}$ d'affilée. On peut, hein, le patient, $7 \mathrm{~h}$ ou $7 \mathrm{~h} 30$ d'anesthésie, il est plus à ça près. Et nous on est dans de meilleures conditions pour opérer de toute façon. Donc je pense que c'est tout bénéf pour le patient. Donc je l'aidais et tout avec un ventre comme ça (elle mime). En tenant les écarteurs comme les autres, comme les mecs et tout. Ça on a fait ça le lundi et on en a refait un le vendredi. Donc la même semaine, la dernière semaine. Et là ça a duré 5 h et j'ai refait l'intervention, j'ai encore aidé. Parce que les autres, y avait eu une greffe de foie, donc y en avait deux qui étaient sur une greffe, quoi. Voilà enfin ça s'est trouvé qu'il fallait que j'y aille, j'y suis allée. Et j'ai tenu aussi le coup. Et c'était la dernière semaine où j'étais bien grosse quand même. Et c'est vrai que quand on voit les infirmières qui sont enceintes et qui, elles, prennent facilement les congés mater, les congés patho et tout ça. Au bout de... 4-5 mois de grossesse (voix un peu plaintive) "Oh le bloc, elles peuvent plus". Déjà le bloc, elles viennent plus aider au bloc, elles font en salle mais elles viennent plus aider... et puis très rapidement elles prennent leurs congés bien plus tôt. Donc quand même il s'est dit : "Ben oui...", que j'avais bien tenu le coup ».
\end{abstract}

Elle accouche au mois de juin, reprend en septembre dans un hôpital périphérique mais ne valide pas son stage puisqu'elle n'y reste que deux mois. Elle fait encore un dernier stage d'orthopédie, mais décide que ce sera son dernier. Ses deux derniers stages se sont très bien passés, elle bénéficie encore de remarques très positives des patrons sur ses rapports semestriels, mais elle dit avoir pris conscience au cours du stage chez le Pr Soquet qu'elle ne souhaite pas continuer la chirurgie. Autrement dit, à ses yeux, ses deux derniers stages résultent de l'inertie de son parcours et correspondent au temps nécessaire à la maturation du projet d'une carrière de spécialiste. Ce fut véritablement le "déclic » du "non je ne veux plus continuer chirurgie. Je veux plus faire chirurgie, je veux plus de cette 
vie-là ». Pourtant, son mari l'encourage à poursuivre, lui rappelant que c'est le seul stage qui se soit mal passé, mais elle est résolue dans sa décision et se réoriente vers la médecine générale, ce qui l'oblige à recommencer depuis le début un troisième cycle, c'està-dire à refaire deux ans et demi d'études ${ }^{17}$.

\section{LE PRIMAT DE LA FAMILLE}

Il apparaît clairement au vu de tout ce qui a déjà été rapporté, que Marie est très « famille ». Ce n'est pas un hasard si les ambiances de stages qu'elle a appréciées sont décrites en insistant sur la dimension familiale des équipes ${ }^{18}$. Elle accorde une grande importance à sa propre famille. Ses choix font passer en priorité son mari et ses enfants sur sa carrière (elle renonce à la gynécologie-obstétrique pour suivre son mari, interrompt son internat par deux grossesses, ne reporte pas la deuxième mais la cumule avec un choix de stage difficile pour ne pas avoir à l'effectuer plus tard avec la charge de deux enfants). Elle m'explique qu'elle a toujours envisagé d'avoir plusieurs enfants: "Je savais que j'en voulais trois de toute façon " et elle aura d'ailleurs sa troisième grossesse pendant son résidanat ${ }^{19}$. Lorsqu'elle m'expose de façon générale pourquoi le rythme de vie et le stress de l'obstétrique est pire que celui de la chirurgie digestive, ses référentiels sont encore ceux d'une vie de famille: "Quand tu as des gamins à la maison qui font pas toujours bien leurs nuits, qui pleurent derrière... c'est pas juste deux nuits de garde où tu dors pas. Voilà, c'est l'horreur». Elle pense que si elle avait continué en chirurgie elle aurait plutôt été en clinique "pour essayer de [s]'aménager un mitemps. Parce qu'avec trois enfants, c'est dur de faire du plein temps. Et à l'hôpital, (elle) pense que c'est difficilement compatible». À la fin de ses deux stages de résidanat, quand une amie, médecin généraliste, lui propose de s'associer avec elle, elle lui demande d'attendre: « D’abord j'ai pas fini mon résidanat, j'ai encore 6 mois et un bébé dans probablement un an, parce que je savais que j'allais le faire. En fait en discutant j'étais tout juste enceinte mais je le savais pas, mais je savais

17/ À partir du moment où elle arrête la chirurgie son mari « a relancé sa carrière à lui, qu'il avait mise entre parenthèses parce qu'il fallait bien que quelqu'un s'occupe des enfants ». Alors qu'il est directeur de cabinet, il s'investit de plus en plus politiquement, devenant attaché territorial et candidat aux législatives pour être député. Elle dit qu'il n'a jamais influé sur sa décision d'arrêter, l'ayant toujours laissée libre de faire ses choix professionnels et l'encourageant à continuer au contraire. Il ne l'aurait pas faite culpabiliser de devoir mettre sa propre carrière politique entre parenthèses. Lui-même aurait poussé ses études jusqu'à un DES parce qu'elle passait le concours de l'internat (elle l'aurait « entraîné » d'une certaine façon).

18/ Elle adopte d'emblée une relation de complicité avec les infirmières sans s'affronter initialement avec elles comme le font la plupart des internes, adoptant d'abord une posture plus masculine (Molinier, 2003).

19/ Troisième cycle des études médicales pour les médecins généralistes, équivalant à l'internat pour les chirurgiens. 
que je voulais un gamin donc... Voilà donc je lui ai dit: "J'arrive pas avant un an". Puis je lui ai dit "honnêtement, avec trois enfants, s'installer à temps plein ça me dit pas tellement”. Moi l'idéal, ce que j'aimerais c'est trouver quelqu'un avec qui m'installer à mi-temps. Mais pour l'instant j'ai pas de piste. Sinon je préfère faire des remplacements, médecine générale, c'est moins contraignant».

Elle invoque "l'instinct maternel » (et naturalise ainsi une disposition socialement construite) pour justifier son choix d'abandonner la chirurgie: "Moi, j'ai mon côté maternel qui fait que j'ai envie d'être à la maison avec les enfants. Mais c'est pas seulement la mentalité qui fait que c'est la femme à la maison, l'homme qui travaille. C'est pas comme ça chez nous. Et pourtant, moi j'ai mon instinct maternel qui veut que je m'occupe des enfants et que j'ai besoin de m'en occuper. J'ai besoin de les voir, de m'en occuper ». Et la conclusion qu'elle en tire est que la famille "c'est pas toujours compatible avec la chirurgie».

Nombre d'enquêtés sont d'accord avec cette dernière affirmation. Mais la grande majorité des femmes chirurgiens rencontrées font passer justement leur engagement professionnel avant leur vie de famille et n'envisageraient pour rien au monde d'arrêter la chirurgie ou de faire un mi-temps pour élever leurs enfants (le mode de garde privilégié par les femmes chirurgiens qui sont mères est la nounou à domicile qui donne le plus de souplesse). La socialisation primaire de Marie ne l'a pas préparée à opter pour une telle priorité et la socialisation chirurgicale a été impuissante pour changer la force de cette inculcation.

\section{DES COMPÉTENCES MAIS UNE APPÉTENCE ET DES DISPOSITIONS CHIRURGICALES QUI FONT DÉFAUT}

Au vu des appréciations portées dans les rapports semestriels qui la concernent, Marie a bel et bien acquis des compétences chirurgicales. Même les appréciations du Pr Soquet vont dans ce sens (et confirment les propos de Marie): "Sera un "bon chirurgien" si elle peut faire l'effort de travailler sur le plan théorique. Était très handicapée par sa grossesse pendant le semestre. A été parfaitement présente et a su dominer sa fatigue ", "doit s'efforcer de lire un peu plus ", " aide parfaitement bien. Douée sur le plan manuel. A été remarquablement présente au bloc opératoire malgré une grossesse avancée. Il faut s'en féliciter ». Lorsque j'interviewe le Pr Soquet et que je lui demande sur quels critères il portait un jugement négatif sur son stage, il 
m'explique que c'est en aucun cas à cause d'un manque de travail : Marie a bien rempli ses fonctions, mais elle "manque de motivation».

En fait, il manque à Marie une chose absolument essentielle en plus des compétences: l'appétence. Alors que les autres candidates à la chirurgie disent avoir choisi la chirurgie par « passion», Marie a fait le choix d'un métier. Or sans l'appétence, les contraintes imposées par le métier s'avèrent trop lourdes pour être supportées durablement. Par ailleurs un certain nombre de dispositions indispensables pour réussir en chirurgie lui font cruellement défaut et justifient sans doute qu'on l'ait un peu malmenée dans le service du Pr Soquet. Lui manquent apparemment le «tempérament chirurgical » (Cassell, 1986), "l'ethos », " la sous-culture chirurgicale» (Katz, 1999) requis.

En effet, le manque d'assurance de Marie contraste avec la confiance conquérante et presque arrogante de la plupart des chirurgiens ${ }^{20}$. Elle ne cesse au cours de l'entretien d'utiliser des phrases dépréciatives à l'égard de ses choix ou de ses goûts: "C'est con mais... », "C'est débile mais...». L'auto-dévaluation est permanente chez elle. Elle rappelle combien elle s'est sous-estimée tout au long de sa scolarité : "J'étais persuadée que j'étais pas capable", "Je pense que j'ai dû leur apporter [aux camarades avec qui elle prépare l'internat et qu'elle décrit comme "intelligents" quand elle se juge laborieuse] mais je me rends pas bien compte». Rappelons qu'elle ne se sent pas capable de prendre un poste de chef de service en urologie pédiatrique, spécialité qui n'est pas la sienne (" J'avais l'impression d'être un imposteur »). D'elle-même, elle affirme: "J'avais pas d'ambition mais (en riant) comme toujours parce que je me sous-estime peut-être. Non, mais je serai pas devenue professeur, c'est sûr j'aurais pas fait ça». Alors que les autres enquêtés en chirurgie ne remettent jamais en question leurs choix, Marie s'interroge et émet quelques regrets : "C'est peutêtre l'erreur que j'ai faite». Si le récit de son parcours en chirurgie est celui d'une «erreur» (l'ayant conduite à revenir en arrière institutionnellement - pour devenir généraliste) et participe sans doute de ce fait à sa posture dépréciative, celle-ci a pu être observée au cours de l'entretien y compris sur des sujets extra-professionnels.

La sensibilité affective exacerbée de Marie est aussi en décalage avec le relatif "endurcissement» exigé en chirurgie. Elle explique au sujet de la gynécologie-obstétrique : "Quand ça se passe mal, c’est

\section{Le manque \\ d'assurance de Marie contraste avec la confiance conquérante et presque arrogante de la plupart des chirurgiens.}

20/ Pearl Katz: "[the surgeon is] an action-oriented male hero who single-handedly performs death-defying feats, acts with certainty, eschews doubts and maintains an even-tempered optimism " (1999, p. 19); Joan Cassell : "The surgeons I observed all seemed to have an overwhelming confidence in themselves and their abilities. (...) an old-time orthopaedic surgeon had once announced: "All surgeons think they're the best; they wouldn't be here otherwise" " (1986, p. 14). 
super dur. Autant en chirurgie, quand on "casse" un malade entre guillemets - c'est un peu l'expression des chirurgiens - quand on "casse" un malade c'est souvent une personne âgée, qui avait un cancer et tout... C'est le risque. On fait ce qu'on peut, bon, le maximum. Mais en obstétrique, on casse un bébé ou une femme jeune. Et là c'est insupportable, quoi, ça c'est..." ", "des choses difficiles à vivre». Elle insiste sur le caractère stressant de cette spécialité. Émotion et stress lui apparaissent difficilement gérables. On est loin de la remarque étonnée de l'interne de chirurgie pédiatrique qui ne comprend pas ma question quand je lui demande : "C'est pas trop dur comme spécialité ? ", ne voyant pas pourquoi opérer des enfants serait plus difficile.

L'attitude d'évitement qui a caractérisé les relations entre Marie et le Dr Luthereau à l'issue de la scène conflictuelle au bloc opératoire sont symptomatiques du manque d'assurance et de l'excès de sensibilité de Marie. Comme on l'a vu, ses co-internes lui conseillent au contraire de "ruer dans les brancards", de "lui rentrer dedans ». D'autres enquêtées rencontrées ont eu effectivement, dans des circonstances analogues, des réactions de face-à-face assez violentes qui se soldent parfois par la nécessité d'une démis$\operatorname{sion}^{21}$. Les réactions de Marie diffèrent fortement de ce mode «frontal». Sa manière de réagir rend davantage compte d'une tactique d'évitement : elle ne lui parle plus, ne l'aide plus au bloc. On peut raisonnablement penser que le Dr Luthereau aurait attendu d'elle d'avoir du répondant (les échanges de "vannes» sont courants dans les rapports entre chirurgiens) et le fait qu'elle ne lui adresse plus la parole ne le gêne aucunement, contrairement à ce que semble croire Marie. En boycottant ce chirurgien, Marie se prive en réalité d'une partie de la formation. Si ce choix d'évitement a parfois été adopté par d'autres enquêtées, les raisons mises en avant par Marie sont bien différentes. En effet, Marie se tait car elle s'estime pour sa part incapable de réagir sans risquer de perdre la face (i.e. de pleurer). Mais intérieurement, elle est minée, profondément atteinte par la situation (elle finira par prendre quelques jours de congés). Les autres enquêtées ayant boycotté des chirurgiens seniors tenant des propos misogynes ont, quant à elles, résolument adopté une attitude de mépris et l'évitement est au contraire le résultat d'une certaine «force morale » au point qu'une d'entre elle, convoquée par le chef de service qui lui conseille de mettre de l'eau dans son vin sans quoi son attitude risque de lui porter préjudice, lui répond sereinement: «on verra bien». 


\section{UNE SOCIALISATION CHIRURGICALE PAS TOTALEMENT RATÉE}

Il serait réducteur de ne voir que les « ratés » dans la socialisation secondaire de Marie. En réalité, sur quelques points au moins, l'internat de chirurgie a contribué à modifier certaines de ses dispositions ou habitudes. Tout d'abord, elle insiste à de nombreuses reprises sur sa très grande timidité avant l'internat. La chirurgie l'aurait véritablement désinhibée dans ses rapports avec les patients adultes (elle se dit à l'aise avec les enfants depuis toujours) comme avec ses collègues. Elle a aussi acquis des habitudes langagières plus relâchées, en rupture avec celles qu'elle a héritées familialement : "Je pense que même dans mon langage, dans ma façon de parler ça doit se retrouver. Je pense que quelque part j'ai des restes. Je dis plus des mots légèrement... pas vulgaires mais je suis plus grossière... Je suis pas soignée dans mon langage. Par exemple je parle de la guirlande, je dis "elle s'est cassée la gueule" au lieu de dire "elle est tombée", quoi. (Avant tu aurais plutôt dit "elle est tombée" ?) Ouais, je pense qu'y a des restes qui doivent venir de là. J'ai un franc-parler plutôt ».

Plus manifeste encore et plus important, elle a véritablement intériorisé l'impératif d'endurance physique ${ }^{22}$ : elle "surmonte sa fatigue » et stigmatise le comportement des infirmières jugées «chochottes " lorsqu'elles prennent des congés pathologiques ou se font remplacer au bloc opératoire. Cette endurance est d'ailleurs honorée par le Pr Soquet dans son appréciation de fin de stage. Mais aussi importante soit-elle, elle apparaît insuffisante pour faire de Marie une candidate sérieuse et désirable en chirurgie.

On peut voir que l'expérience du scoutisme a sans doute contribué à la préparer à ces aspects de la chirurgie. Elle explique en outre que le fait d'avoir été cheftaine et de prendre des responsabilités l'a aidée professionnellement à trouver de l'assurance. Si sa socialisation familiale l'a plutôt préparée à un rôle de femme, l'expérience socialisatrice du scoutisme est une influence hétérogène qui complexifie la socialisation primaire de l'enquêtée et peut expliquer une orientation professionnelle qui apparaît improbable. 


\section{«C'ÉTAIT UN SERVICE À RENDRE A LA PROFESSION »}

Peter L. Berger et Thomas Luckmann, lorsqu'ils évoquent le troisième cas de "socialisation ratée », expliquent qu'un des obstacles à la réussite de la socialisation vient de la résistance du milieu luimême: "La structure sociale ne permet pas à l'individu la réalisation de l'identité subjective choisie ». Si Marie présente son parcours et sa reconversion professionnelle comme pleinement choisis, on peut s'interroger et se demander si celle-ci n'est pas plutôt le résultat d'une éviction collectivement initiée par ses pairs. Notre propos n'est pas de dire qu'elle a été « savamment orchestrée ». Mais de fait, les interactions répétées avec plusieurs praticiens ayant des attitudes et façons de penser convergentes ("pousser l'interne dans ses retranchements », « être volontaire », « être ambitieux »), mais opposées en grande partie aux siennes, « usent » littéralement Marie au point qu'elle a l'impression d'endosser librement sa démission chirurgicale. En réalité, incapable de s'adapter, elle offre la preuve à ses supérieurs hiérarchiques qu'ils ont eu raison de ne pas croire en elle. Dans ces conditions, ils sont tous simplement heureux " pour elle», et surtout pour la profession, de la voir renoncer.

Le Dr Luthereau et le Pr Soquet, interrogés indirectement sur le cas de Marie (ils ignorent que je suis en contact avec elle et que je mets un nom sur ce «cas»), reconnaissent tous deux avoir un peu été « durs» avec elle. Toutefois, ils justifient leur attitude par les lacunes dispositionnelles dont elle fait preuve (manque d'assurance, de distanciation, d'ambition) et qui prouvent que finalement « elle n'est pas faite » pour être chirurgien (sa démission étant l'ultime preuve confirmant leur jugement).

EZ : Au sujet des livrets de stage, j'ai appris qu'il y avait une appréciation des chefs de service. On m'a dit que le stage était toujours validé.

Pr Soquet : Euh... En gros, à part quelques exceptions. C'est arrivé que je valide pas. Presque toujours validé. Parce que... Par contre, j’ai eu quelquefois à la fin du stage des réunions très dures avec certains externes pour leur expliquer qu'il fallait probablement qu'ils choisissent une autre spécialité, que la chirurgie.

EZ : C'était... des profils qui collaient pas?

Pr S : Deux fois. Y en a un qui s'est orienté vers la psychiatrie. Et puis y a une fille qui a abandonné la chirurgie. Elle était pas faite pour ce métier. (...) Y en a un qui n'était pas fait pour la chirurgie, il n'avait pas d'esprit de décision, il avait un esprit d'analyse très poussé, et il perdait beaucoup de temps, il avait pas... il était pas... il avait pas le tempérament d'un chirurgien. Et la fille en question, elle avait pas de motivation. Aucune motivation. Donc je pensais que ça serait un mauvais chirurgien. 
EZ: (...) Alors vous avez quand même validé leur stage d'après ce que vous dites?

Pr S: J'ai validé parce qu'ils avaient quand même rempli leur travail correctement, ils avaient pas été incorrects du tout. Il m'a semblé que je leur rendais plutôt service en leur disant qu'il fallait - ils étaient encore jeunes - s'orienter vers une autre spécialité.

EZ: Est-ce qu'ils ont eu une mauvaise appréciation et que le stage a quand même été validé?

Pr S: J'ai pas mis une mauvaise appréciation. Parce que j'avais pas à leur reprocher... une qualité de travail. J'avais à leur... J'ai pensé que ça serait peut-être pas des... C'était un service à leur rendre, et à rendre à la profession en tout cas, que de les orienter vers une autre spécialité que la chirurgie.

Si le candidat à la chirurgie choisit un métier, la profession a le pouvoir de sélectionner et d'élire ceux qu'elle juge dignes de reproduire le corps professionnel. Marie n'apparaissait pas comme une candidate désirable, le Pr Soquet se charge de le lui dire. Marie ne s'y trompe pas, quand bien même l'instance socialisatrice chirurgicale lui envoie deux messages contradictoires: "Vous n'êtes pas faite pour ce métier » (ce que le Pr Soquet lui dit ouvertement en fin de stage, ce qu'indirectement on ne cesse de lui faire sentir au cours du stage) et «fera un bon chirurgien si elle change certaines choses » (l'appréciation est plutôt positive, on valide son stage et l'admet à poursuivre sa formation ${ }^{23}$ ) : elle n'était pas à sa place en chirurgie et n'est pas portée par l'institution. Finalement, le choix final de poursuivre ou d'abandonner dans cette voie est laissé à la candidate elle-même. La socialisation secondaire était en cours. Les chirurgiens admettent bien que sa formation soit inachevée et l'ont "poussée dans ses retranchements » espérant qu'elle adopte finalement des dispositions plus chirurgicales. L'abandon de Marie est interprété de manière naturalisante (" elle n'était pas faite pour ce métier ») tandis qu'il faut voir là une prégnance de la socialisation primaire, manifestement insurmontable tant le décalage avec les valeurs prônées en chirurgie était grand pour Marie.

\section{CONCLUSION}

Certaines dimensions de la socialisation primaire de Marie ont sans aucun doute été un handicap majeur pour intégrer le milieu chirurgical. Éduquée dans un milieu familial prônant le schéma traditionnel de la répartition sexuée entre les rôles, elle se

23/ Il est courant que les chefs de service valident un semestre insatisfaisant, ne serait-ce que parce qu'ils sont tenus sinon de reprendre l'étudiant pour un deuxième stage et qu'ils ne souhaitent pas garder un étudiant ne donnant pas toute satisfaction.

\section{La profession a le pouvoir de sélectionner et d'élire ceux qu'elle juge dignes de reproduire le corps professionnel. Marie n'apparaissait pas comme une candidate désirable.}


construit comme une "petite fille» des classes supérieures et intègre durablement le primat de la famille dans ses choix professionnels. Si au cours de sa formation Marie acquiert manifestement des compétences réelles en chirurgie (notamment techniques) et intériorise des types de comportements valorisés dans la discipline (franc-parler, endurance physique), sa socialisation chirurgicale reste incomplète. Elle continue d'afficher une sensibilité exacerbée, un manque d'assurance et surtout d'ambition. Enfin, elle manque cruellement d'appétence pour la chirurgie comme discipline: son orientation tient davantage à son rang de classement qui rendait difficile de ne pas opter pour une voie considérée comme prestigieuse. Ces lacunes dispositionnelles sont révélées au cours du semestre chirurgical le plus exigeant de tout son internat. Dans la mesure où ce service hospitalier très réputé incarne au plus haut point les valeurs chirurgicales, le décalage entre les dispositions personnelles de Marie et les exigences comportementales et intellectuelles que le milieu exige d'elle est alors maximal. En « décidant» de mener une grossesse au cours de ce semestre essentiel de sa formation, elle ne fait que témoigner de son «manque de motivation » (puisqu'elle donne sa priorité à la famille) quand tous les chirurgiens du service estiment comme un devoir de faire passer le métier avant tout. Son abandon et sa reconversion professionnelle, initiés par le milieu lui-même qui ne cesse de souligner ses lacunes pour l'inciter à y remédier rapidement, sont finalement l'ultime preuve qu'elle n'était «pas faite» pour ce métier. En réalité, le décalage entre socialisation primaire et socialisation secondaire était si grand qu'elle renonce à poursuivre la seconde. Le parcours de Marie vient confirmer la prégnance de la socialisation primaire. Mais si l'analyse s'arrêtait là on ne verrait au fond qu'un cas de «socialisation féminine réussie ». Or il faut voir aussi en quoi ce portrait est révélateur de la socialisation chirurgicale.

En creux, ce qui ressort de ce portrait, ce sont les valeurs chirurgicales elles-mêmes, portées par l'institution et incarnées par ses membres: ténacité, assurance, endurance physique et morale, sens de la répartie, franc-parler. Autant de valeurs socialement construites comme masculines et qui découragent nombre de femmes d'intégrer ce milieu (expliquant ainsi que la chirurgie ait le plus faible taux de féminisation de toutes les spécialités médicales ${ }^{24}$ à l'heure où la médecine est désormais largement féminisée ${ }^{25}$ ). Ce qu'il faut du coup

24/ Autour de $10 \%$ de femmes pour la chirurgie générale et digestive, environ $3 \%$ pour l'urologie et l'orthopédie.

25/ Les filles représentent plus de $60 \%$ des jeunes promotions d'étudiants en médecine à l'heure actuelle 
se demander pour les candidates à la chirurgie, c'est ce qui, dans leur socialisation primaire, permet d'envisager une telle orientation et comment leurs dispositions et leur appétence leur permet de «tenir » dans ce milieu masculin, particulièrement soucieux de sa reproduction sociale.

Emmanuelle Zolesio

GRS Lyon II, ENS-LSH, Universités de Lyon ezolesio@ens-lsh.fr

\section{RÉFÉRENCES BIBLIOGRAPHIQUES}

BERGER P. L., LUCKMANN T., La construction sociale de la réalité. Paris : Armand Colin. 2006 [1966]

BREUIL-GENIER P., SICART D., L'origine sociale des professionnels de santé. Études et 2006

CASSELL J., 1986

DREVILLON E., 2003

GLASER Barney G., STRAUSS

Anselm L., 1995 [1967]

HARDY-DUBERNET A.-C., 2000

Résultats : DREES, no 496, p. 1-8.

Dismembering the Image of God : Surgeons, Wimps, Heroes and Miracles. Anthropology Today, vol. 2, n 2, p. 13-15.

Professeur Leca. Chirurgien du cœur. Paris : Éditions Anne Carrière.

La production de la théorie à partir des données, Enquête, Les terrains de l'enquête, mis en ligne le 7 janvier 2008. URL : http://enquete.revues.org/document282.html

Faire (quelle ?) médecine... À propos de la loi de 1982 sur la réforme du troisième cycle des études médicales in Schweyer F.-X., Cresson G. (dir.), Professions et institutions de santé face à l'organisation du travail. Aspects sociologiques. Paris : Éditions ENSP, p. 87-96.

HARDY-DUBERNET A.-C., 2003 L'internat de médecine ou la formation par la concurrence, in Cresson G., Drulhe M., Schweyer F.-X. (dir.), Coopérations, conflits et concurrence dans le système de santé. Paris : Éditions ENSP, p. 75-87.

KATZ P., 1999

The scalpel's edge. The culture of surgeons. Allyon and Bacon : Boston.

LAHIRE B., 2002

Portraits sociologiques. Dispositions et variations individuelles. Paris : Nathan.

LASVERGNAS I., 1986

Le corps étranger ou la place des femmes dans l'institution scientifique. Thèse de sociologie (Phd), Université de Montréal.

MOLINIER P., 2006 [2003]

L'énigme de la femme active. Égoïsme, sexe et compassion, Paris : Petite Bibliothèque Payot, $266 \mathrm{p}$

MARRY C., 2004

Les femmes ingénieurs. Une révolution respectueuse. Paris : Belin. 
Emmanuelle Zolesio

QUEMIN A., 1998

ZOLESIO E., 2004
Les femmes commissaires-priseurs. Sociétés Contemporaines, $\mathrm{n}^{\circ} 29$, p. 87-106

Socialisation professionnelle des chirurgiens, DEA de sociologie sous la dir. de B. Lahire, Université Lyon II. 